\title{
Low Cost Beam-Steering Approach for a Series-Fed Array
}

\author{
Nicholas K. Host, Chi-Chih Chen, and John L. \\ Volakis \\ The Ohio State University \\ Columbus, USA
}

\author{
Félix A. Miranda \\ NASA John Glenn Research Center \\ Cleveland, USA
}

\begin{abstract}
Phased array antennas showcase many advantages over mechanically steered systems. However, they are also more complex and costly. This paper presents a concept which overcomes these detrimental attributes by eliminating all of the phased array backend (including phase shifters). Instead, a propagation constant reconfigurable transmission line in a series fed array arrangement is used to allow phase shifting with one small $(\leq 100 \mathrm{mil})$ linear mechanical motion. A novel slotted coplanar stripline design improves on previous transmission lines by demonstrating a greater control of propagation constant, thus allowing practical prototypes to be built. Also, beam steering pattern control is explored. We show that with correct choice of line impedance, pattern control is possible for all scan angles. A 20 element array scanning from $-25^{\circ} \leq \theta \leq$ $21^{\circ}$ with mostly uniform gain at $13 \mathrm{GHz}$ is presented. Measured patterns show a reduced scan range of $12^{\circ} \leq \theta \leq 25^{\circ}$ due to a correctable manufacturing error as verified by simulation. Beam squint is measured to be $\pm 2.5^{\circ}$ for a $600 \mathrm{MHz}$ bandwidth

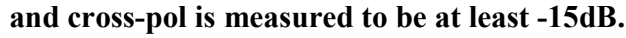

Keywords - Phased arrays, phase control, transmission line, patch antennas, propagation constant

\section{INTRODUCTION}

Phased array antennas allow for many advantages over traditional mechanically steered systems. By electronically positioning the beam, phased array antennas allow for quick beam steering without the hindrance of vibration or overshoot. However, this increased capability comes with a large increase in cost and complexity. The high cost of phased arrays prevents their use in many applications, especially those with high gain requirements since price increases with number of elements. This high price originates from the backend where much circuitry is required to induce beam steering. Most notably, a phase shifter is required for every element in the array.

Many techniques are employed to reduce the cost of phased arrays such as array thinning [1] and sub-arraying [2]. However, these techniques reduce the effectiveness of the array and only moderately reduce the cost. Instead, our approach employs a novel feeding network to eliminate the costly backend. We use a series fed array utilizing a wave velocity reconfigurable transmission line to induce steering [3]. Rather than use a phase shifter at each individual element we use one reconfigurable transmission line to deliver the

This work was supported in part by the NASA Office of the Chief Technologist's Space Technology Research Fellowship (NSTRF), NASA Grant \#NNX11AN16H correct phase to every element.

This paper presents a slotted Coplanar Stripline (CPS) transmission line with a tapered dielectric insert positioned between the two line traces [4]. The wave velocity is then controlled by varying the portion of the insert to which the transmission line field is exposed. Importantly, the profile of the insert can be shaped to give the desired propagation constant control.

In our design, we eliminate the need for phase shifters and instead mechanically modify ( $\leq 100 \mathrm{mil}$ linear motion) the transmission line for phase shifting. Several other approaches use reconfigurable transmission lines to induce beam steering [5-10]. In [5], a nonfoster circuit is used at each element to realize true time delay at the expense of complexity and cost. Others use ferrite [6-8] which can be biased to change the electrical properties. However, the use of ferrite adds excessive weight and puts an inherent cap on bandwidth. More complex solutions have also been proposed such as the use of fluid within the transmission line [9]. This, however, is only marginally effective. Finally, [10] uses a concept similar to ours by positioning a dielectric sheet close to the microstrip line in a corporate feeding scheme. However, this setup is bulky. Our approach offers a lightweight, simple, cost effective alternative.

\section{OPERATION PRINCIPLES}

\section{A. Transmission Line Propagation Constant Reconfigurability via a Small Mechanical Movement}

A slotted CPS transmission line with a tapered dielectric insert positioned between the line traces [4] (depicted in Figure 1) is employed in a series fed array topology to give more control over the line propagation constant as seen in Figure 2. By positioning various portions of the tapered insert between the CPS traces, the propagation constant can be controlled. Importantly, the profile of this insert can be shaped to give a desired beam steering response (i.e. a taper to give a linear scan angle to $g$ relationship). For simplicity we have constrained the profile to a linear edge. 


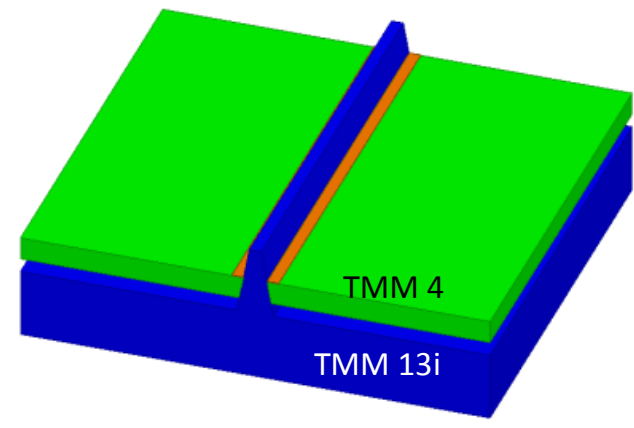

Fig. 1. Reconfigurable slotted CPS transmission line for greater propagation constant control
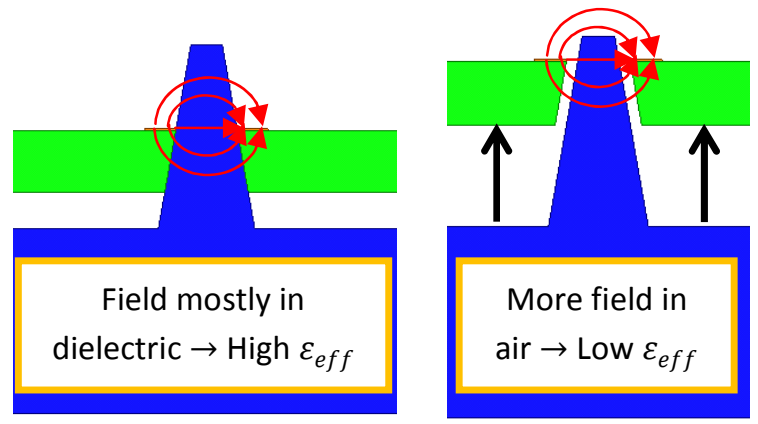

Fig. 2. Insert movement to control CPS propagation constant

As changes in the effective dielectric constant are introduced by reconfiguring the transmission line, the progressive phase delivered to the array elements is also altered, inducing beam steering. Reconfiguring the transmission line also leads to a change in characteristic impedance. This will be addressed in section F. The scan angle achieved through reconfiguration is a function of both effective propagation constant and element spacing. Figure 3 shows the scan angle for propagation constant and element spacing. Specifically, the propagation constant is given as a normalized value to avoid frequency dependency $\left(\mathrm{k}_{\mathrm{eff}} / \mathrm{k}_{0}=\right.$ $\varepsilon$ eff $\mu$ eff $\cong \varepsilon$ eff.

The information in Figure 3 allows for an understanding of the necessary propagation constants given an element spacing and desired scan range. For instance, a half wavelength array scanning from $-30^{\circ} \leq \theta \leq 30^{\circ}$ would require a propagation constant $1.5 \leq \mathrm{k}_{\mathrm{eff}} / \mathrm{k}_{0} \leq 2.5$. Notice that scan angle decreases for a given propagation constant as the element spacing is decreased. This makes physical sense since as the distance between elements decreases, the progressive phase has less transmission line length to accumulate. Additionally, there are multiple ways to scan to a desired angle for a given element spacing. This is due to the cyclic nature of the wave; one can get the desired scan angle by the standard phase or by the standard phase plus $2 \pi$.

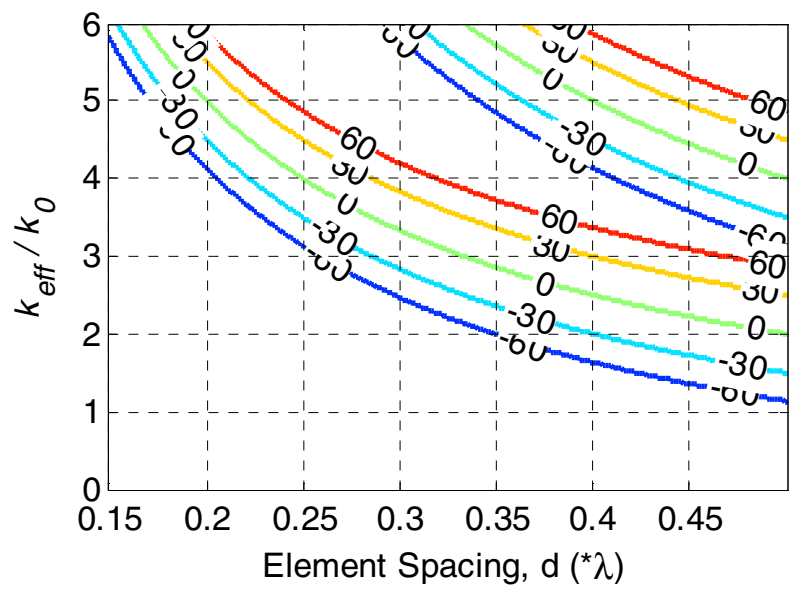

Fig. 3. Scan angle from boresight as a function of propagation constant and element spacing

\section{B. Insert Angle Effect}

There are several key parameters for the CPS line (as seen in Figure 4). We note that the insert is TMM13i, the highest permittivity board from Rogers, thus giving the highest max propagation constant. The first parameters to be explored are the dielectric used for the array board (green) and angle of insert shown in Figure 5. Comparing $\alpha=5^{\circ}$ to $10^{\circ}$ (solid, dashed respectively) one can see that the larger angle can reach lower propagation constants than the smaller angle. This is expected, since when the insert is retracted, it will leave a larger hole. The larger angle also falls more quickly from its max value. For the larger angle, more air is introduced for the same change in $\mathrm{g}$. This quick falling from the max value is the undesirable quality this design is trying to avoid. The great sensitivity of propagation constant in those regions necessitates restrictive tolerances in prototype assembly.

\section{Array Board Dielectric Effect}

The second parameter to consider is the dielectric used for the array board. As expected, the higher the permittivity, the higher the propagation constant. The effect is disproportionally more important in the low propagation constant region. This is because the high permittivity insert is dominant when positioned completely in. Characteristic impedance is also displayed in Figure 5. As expected, as the propagation constant lowers (effective permittivity lowers), the characteristic impedance increases.

\section{Strip Spacing Effect}

The next key parameter to consider is the strip spacing, S. Figure 6 reveals that for wider strip spacings, the propagation constants achieved are larger. Wide strip spacings allow for the high dielectric insert to be wider. Additionally, The propagation constant for both cases drops off at the approximately same rate as $g$ is increased. This is because both cases have the same insert angle. When the insert is retracted, the same amount of air is introduced for both cases. Impedance, again, is a result of the inverse relationship with effective permittivity.

\section{E. Trace Width Effect}

The final key parameter to consider is the trace width, W. Figure 7 depicts the propagation constant and impedance vs. 
gap, g. As the trace width increases, the maximum propagation constant decreases and the minimum propagation constant increases. This is a consequence of the fringe fields of the transmission line. With a wider trace, the field becomes less concentrated between the two conductors. In the high propagation constant region it means more fringe field in air, while in the low propagation constant region it means more fringe field in the array board. Initially it seems that a thinner trace will always be better. However, trace width is also the main determinant of the characteristic impedance. By changing $\mathrm{W}$, the line characteristic impedance is drastically altered.

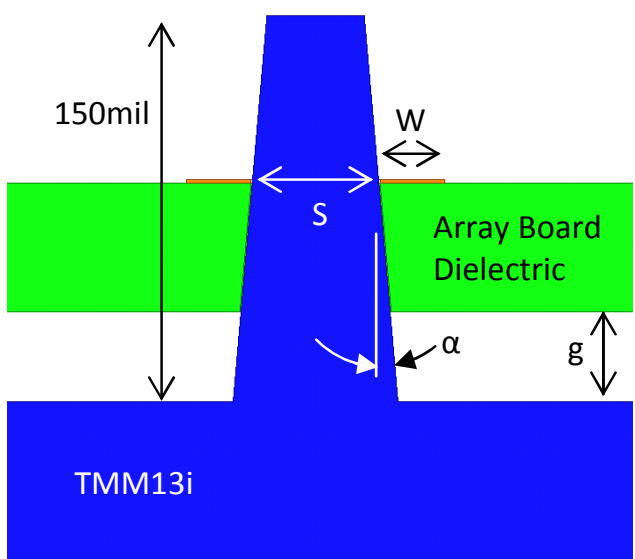

Fig. 4. Key parameters of CPS line

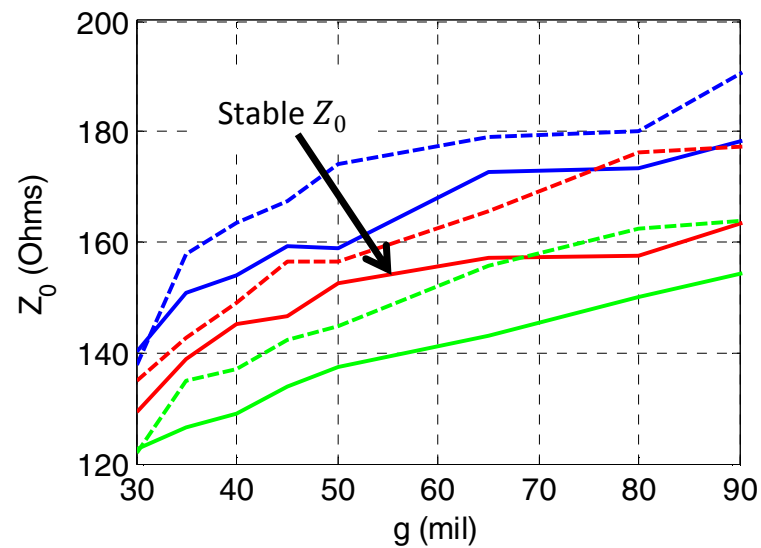

Fig. 5. Propagation constant (left) and characteristic impedance (right) for $\mathrm{S}=50 \mathrm{mil}, \mathrm{W}=25$
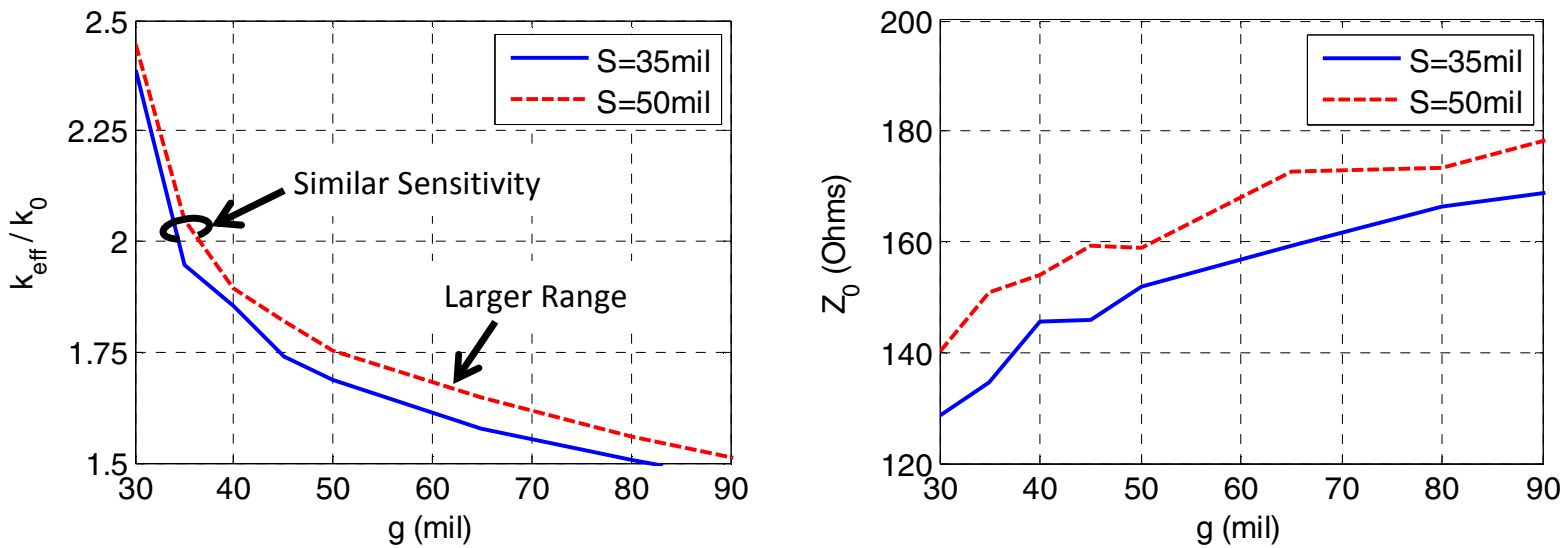

Fig. 6. Propagation constant (left) and characteristic impedance (right) for TMM3, W=25 

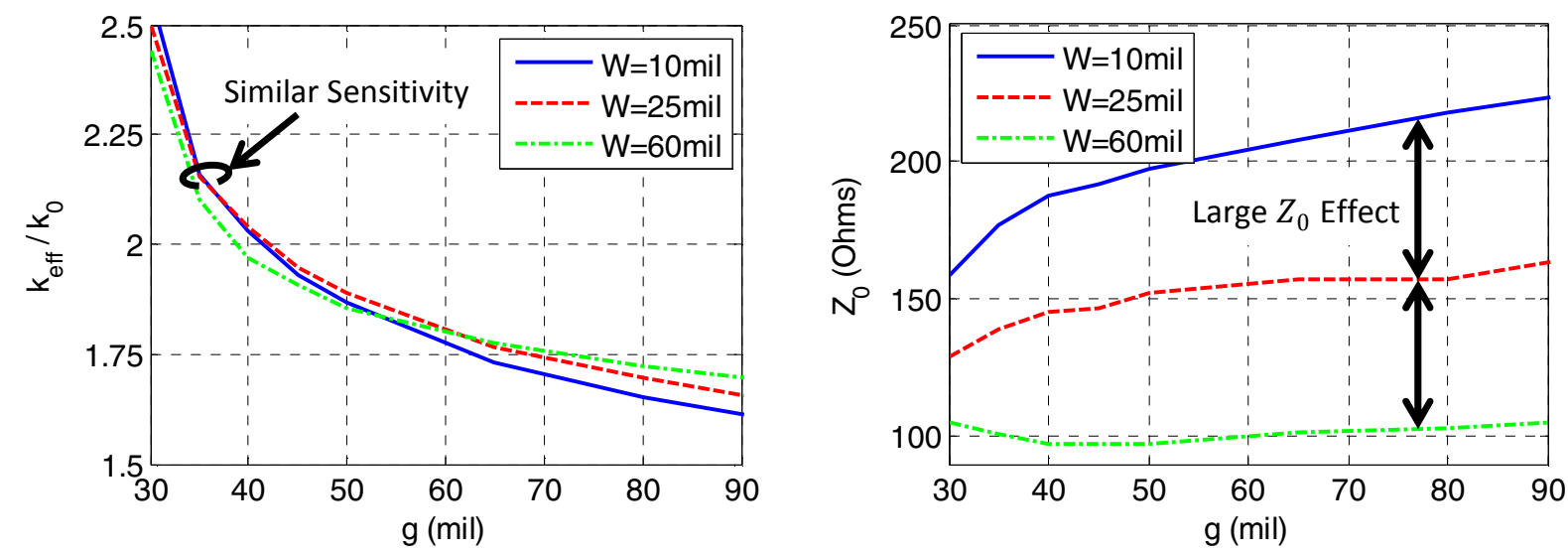

Fig. 7. Propagation constant (left) and characteristic impedance (right) for TMM4, $\mathrm{S}=50 \mathrm{mil}$

\section{F. Array Pattern Control via Uniform Excitation}

In addition to inducing scanning, reconfiguring the air gap also changes the transmission line characteristic impedance. Achieving perfect match over a range of scan angles is challenging. To begin, we will aim to deliver equal excitation amplitude to each element and also ensure perfect match at the feed. Figure 8 depicts the array represented as a circuit. Here, $\mathrm{Z}$ is used to denote the element impedance, $\mathrm{d}$ the element spacing, $\mathrm{k}$ the effective propagation constant, and $\eta$ the line impedance.
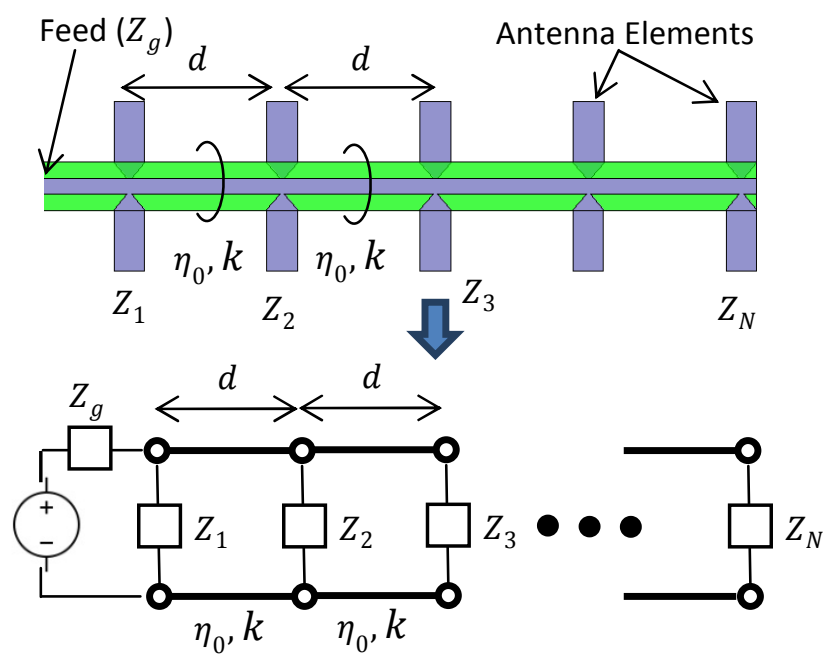

Fig. 8. Schematic and circuit representation of the phased array concept

As derived in [3], to achieve equal amplitude distribution, the ideal impedance taper is given as

$$
Z_{p+1}=\eta_{0}\left(\frac{z_{p} \cos (k d)-j(N-p) \eta_{0} \sin (k d)}{\eta_{0} \cos (k d)-j \frac{Z_{p}}{N-p} \sin (k d)}\right)
$$

The entire array must also be matched to the generator impedance, $\mathrm{Z}_{\mathrm{g}}$. We pick the line impedance equal to the generator impedance. Combining with the uniform excitation requirement, this gives

$$
\mathrm{Z}_{1}=\mathrm{N}_{0}
$$

\section{G. Scanning Effect on Required Element Impedance Distribution}

We now consider when the same transmission line is used between every element. In general, as the array is scanned ( $\mathrm{k}$ is varied) the required impedance profile changes. Figure 9 shows this taper for various scan angles. One observes that the ideal taper substantially varies as the scan angle is changed, indicating that optimizing for any one scan angle is insufficient.

However, by constraining the $\vartheta=30^{\circ}$ scan angle element impedance taper to be linear, the surrounding scan angles converge on this linear taper. The linear taper is accomplished through the correct choice of line impedance. We note that this is not a simple task since characteristic impedance is a function of gap. However, with careful line design, fluctuation can be minimized. Equation 3 gives the necessary choice of characteristic impedance.

$$
\eta_{0}=\frac{\mathrm{NZ}_{\mathrm{g}}}{\sqrt{2}(\mathrm{~N}-1)} \sqrt{1+\left(\frac{\mathrm{N}-2}{\mathrm{~N}-1}\right)^{2}}
$$

Figure 10 depicts the ideal impedance taper when equation 3 is employed. The convergence is further improved by increasing the number of elements as seen in Figure 11. 

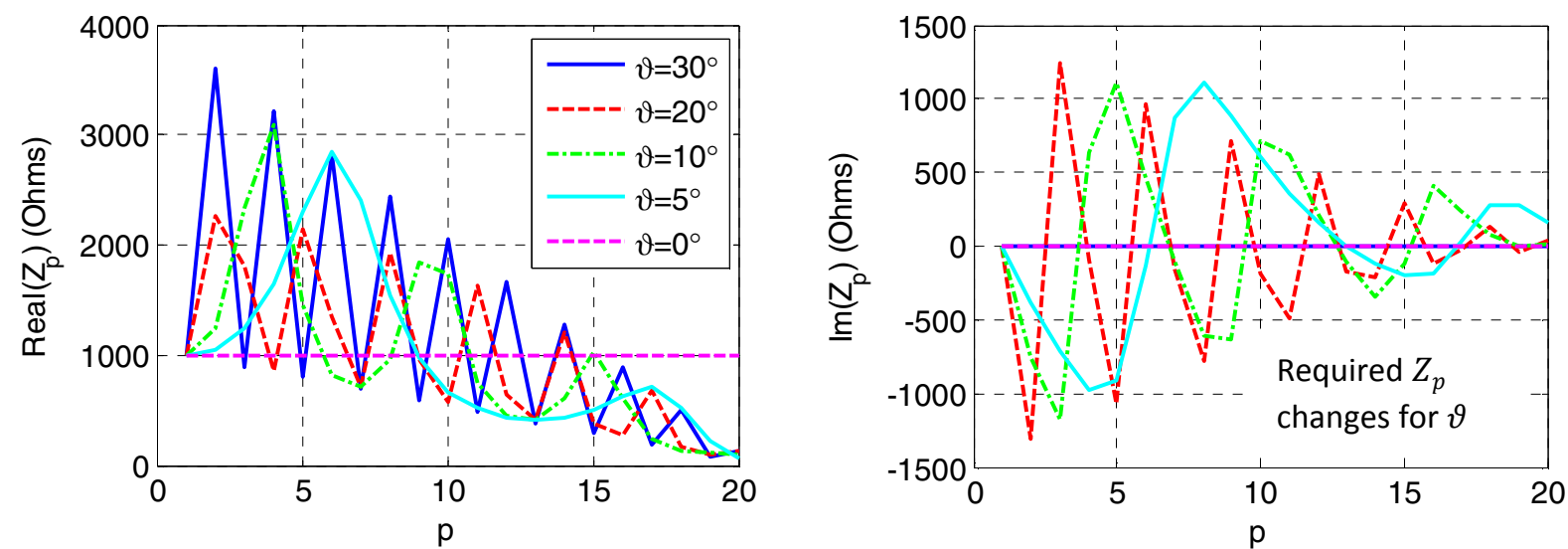

Fig. 9. Required element impedance taper for uniform excitation with $Z_{g}=50 \Omega, d=\lambda / 2, \eta_{0}=100 \Omega, N=20$, and $k$ varied for different scan angles
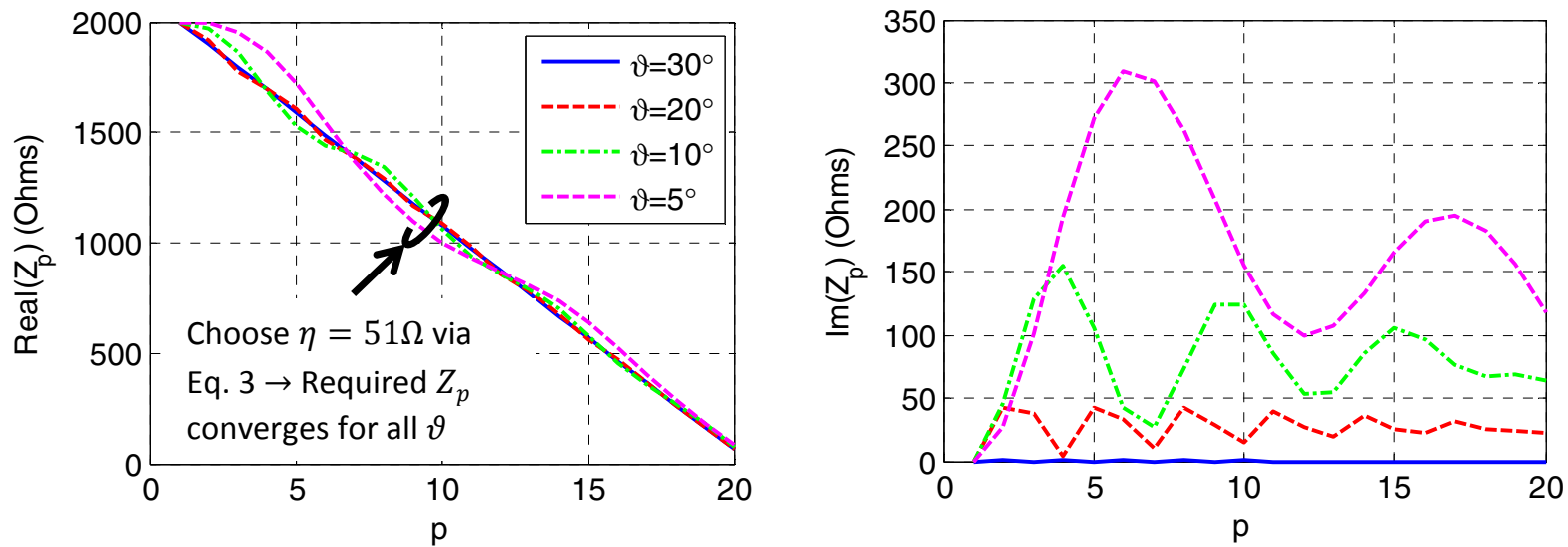

Fig. 10. Required element impedance taper for uniform excitation with $\eta_{0}=51 \Omega$ chosen from Eq. $3, Z_{g}=\eta_{0}, d=\lambda / 2, N=20$, and $k$ varied for different scan angles
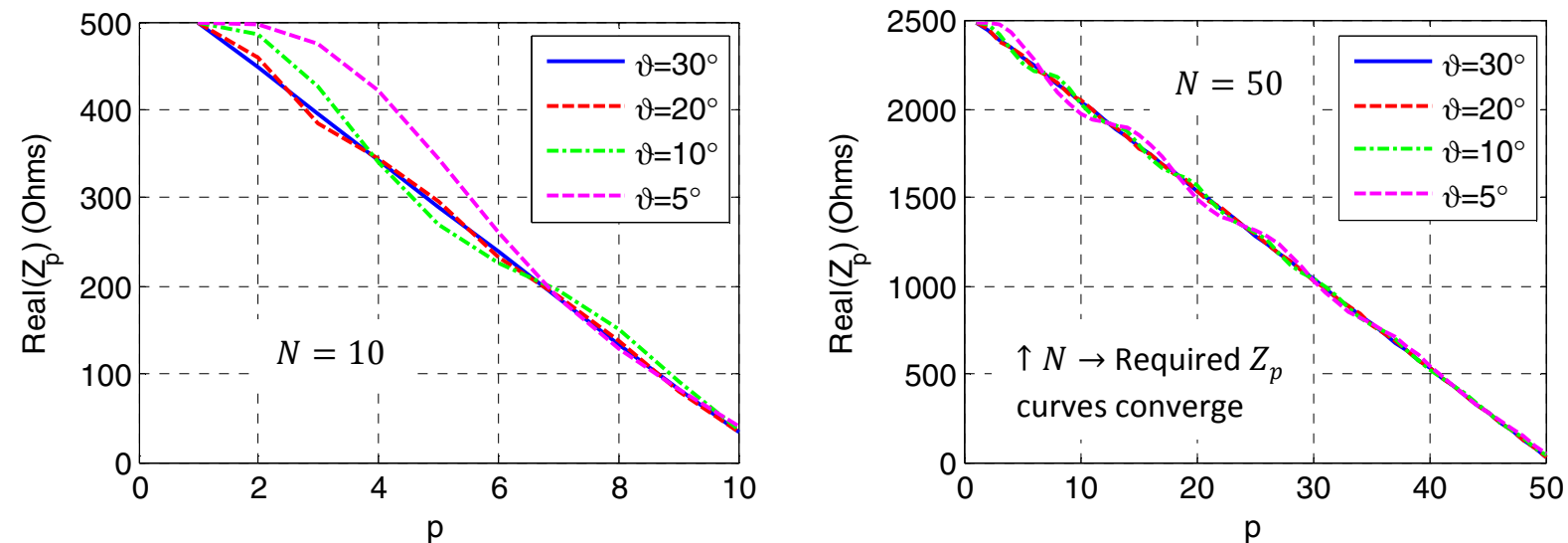

Fig. 11. Required element impedance taper for uniform excitation with $\eta_{0}$ chosen from Eq. $3, Z_{g}=\eta_{0}, d=\lambda / 2, k$ varied for different scan angles, and $N=10$ (left) or $N=50$ (right) 


\section{FINAL SLOTTED CPS ARRAY}

\section{A. Physical Specifications}

As a compromise between scan range and decreased sensitivity, the final CPS design uses TMM4, $\alpha=5^{\circ}$, $S=50 \mathrm{mil}$, and $W=25 \mathrm{mil}$. Employing this transmission line, an array of patches was designed to operate at $13 \mathrm{GHz}$ as seen in Figure 12. Patches were chosen over dipoles due to the easily controllable impedance via feed inset. Additionally, dipoles are not well suited for this application due to their weak radiation when fed by our CPS line. A linear change in patch inset, maximized at the first element and no inset on the final element, is used to achieve close to uniform power distribution. Additionally, a balun design from [11] is used to feed the array.

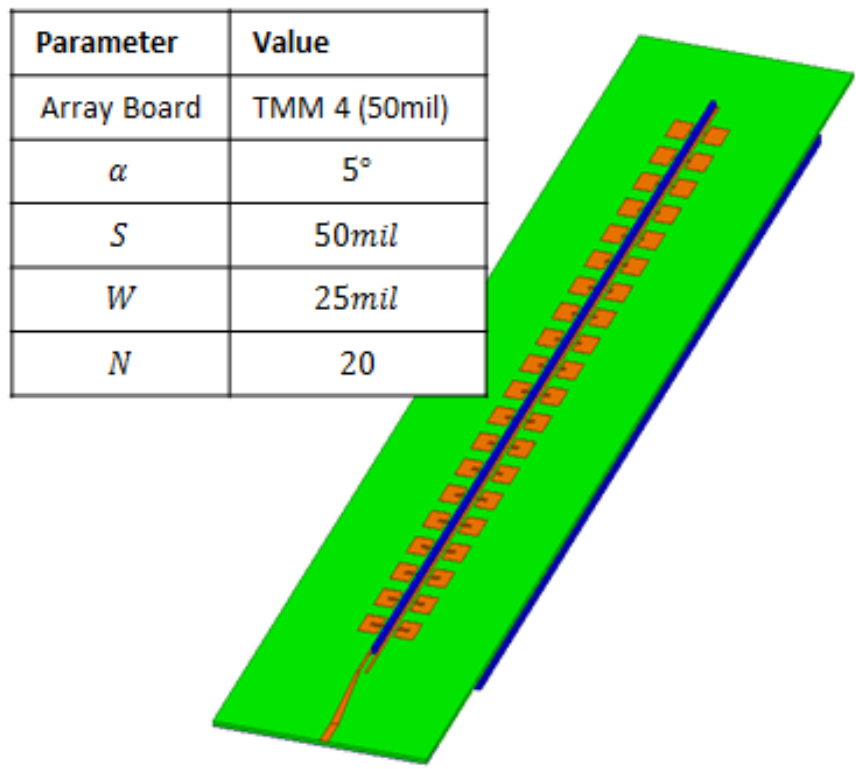

Fig. 12. CPS patch array design employing a tapered patch inset length along the array

\section{B. Simulated and Measured Beam Steering Performance}

The linear inset taper is not an optimized design but is well suited to demonstrate the beam steering ability of the array. Figure 13 shows the scan patterns of the array. Beam steering with mostly uniform gain is achieved from $-25^{\circ} \leq \theta \leq 21^{\circ}$. In addition, this is achieved with a gap of $30 \mathrm{mil} \leq \mathrm{g} \leq 80 \mathrm{mil}$ instead of the $0 \mathrm{mil} \leq \mathrm{g} \leq 9 \mathrm{mil}$ the parallel plate array (as seen in [3]) requires. This desensitization allows for the formation of a pattern even if there is some fluctuation in the propagation constant along the line.

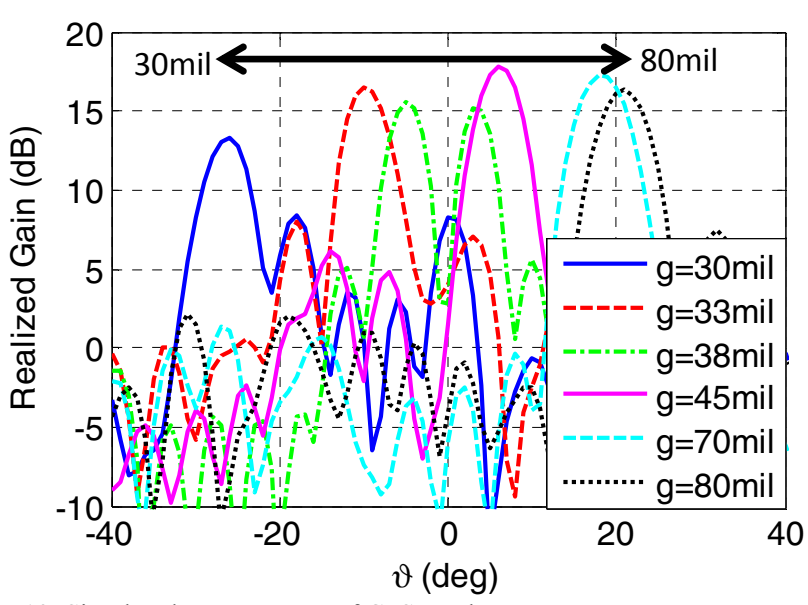

Fig. 13. Simulated scan patterns of CPS patch array

Figure 14 shows the assembled prototype. During assembly, the top board broke in two pieces. This was fixed by gluing it back together and adding on nylon rods as bracers. Binder clips were used instead of traditional clamps for their consistent pressure applied as well as for ease of assembly and disassembly.

Figure 15 depicts the measured co-polarization scan patterns garnered from the prototype. Figures 16, 17, and 18 show the cross-polarization patterns, $\mathrm{S}_{11}$, and beam squint respectively. Cross polarization is about $-15 \mathrm{~dB}$ down compared to co-polarization, $\mathrm{S}_{11}$ is high due to a fractured board, and for a bandwidth of $600 \mathrm{MHz}$, a high communications bandwidth, the beam shifts $\pm 2.5^{\circ}$.

Comparing the measured scan patterns (Figure 15) to the expected scan patterns (Figure 13), there are two differences; reduced gain and reduced scan range. The reduced gain can be attributed to the broken feed structure. This was confirmed via a time domain $S_{11}$ measurement.

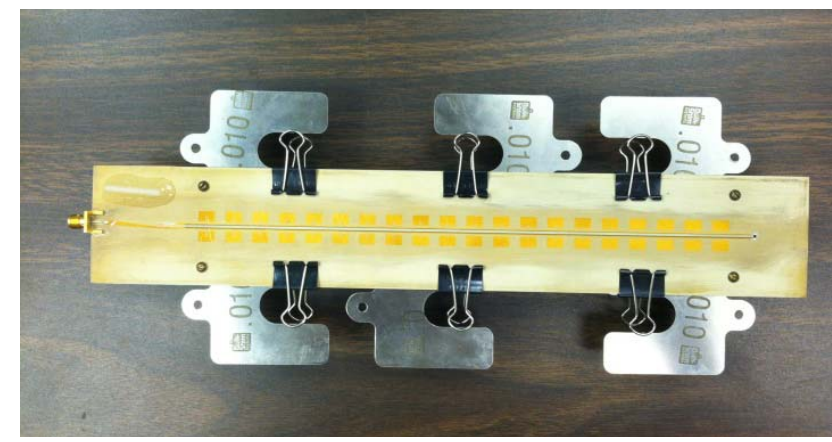

Fig. 14. Assembled prototype of CPS patch array 


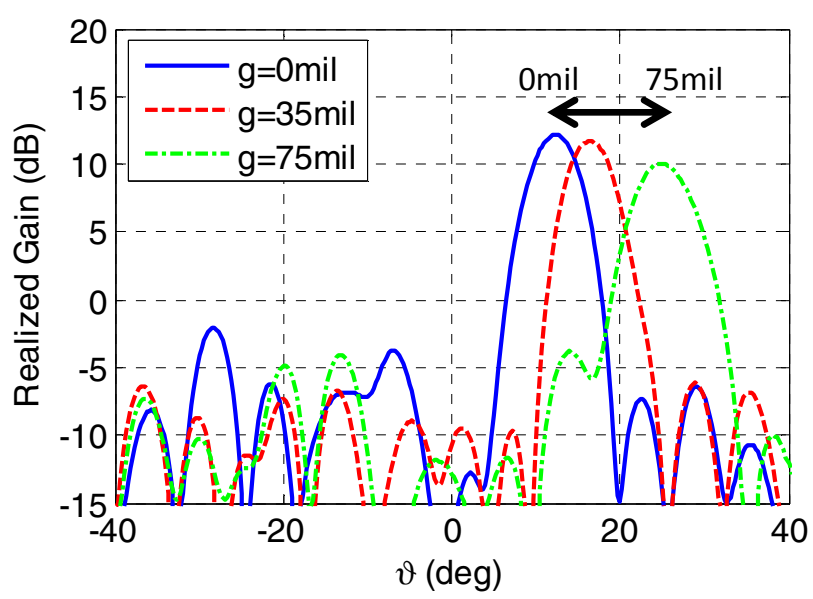

Fig. 15. Measured co-polarization scan patterns of CPS patch array

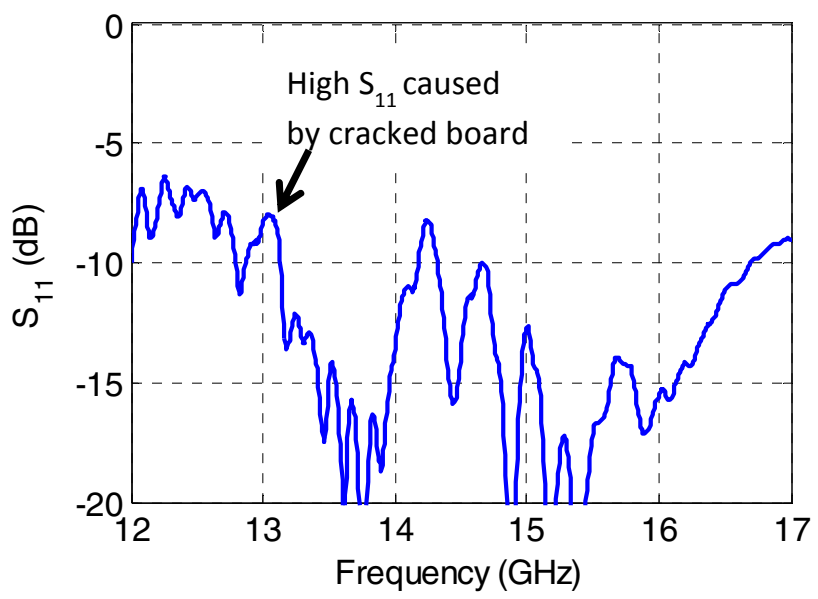

Fig. 16. Measured $\mathrm{S}_{11}$ at $\mathrm{g}=35 \mathrm{mil}$, increased due to a fractured board

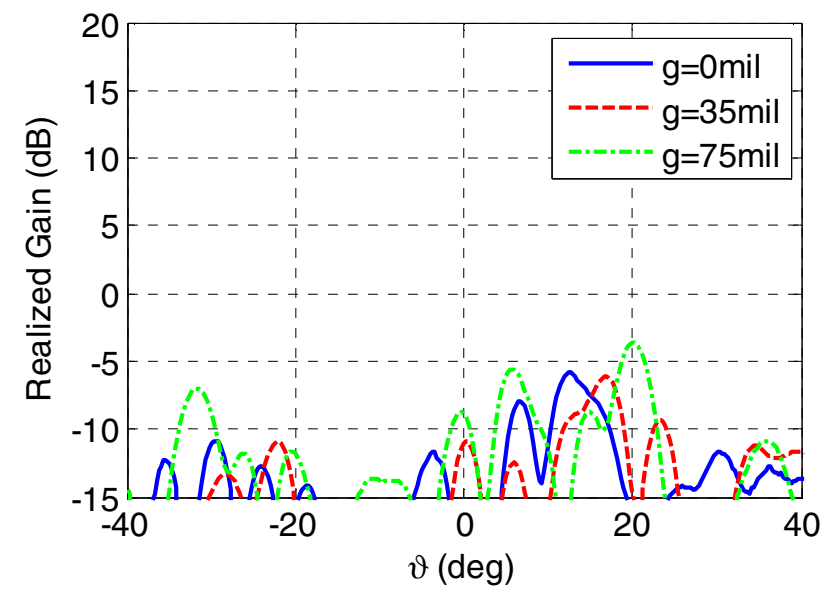

Fig. 17. Measured cross-polarization scan patterns of CPS patch array

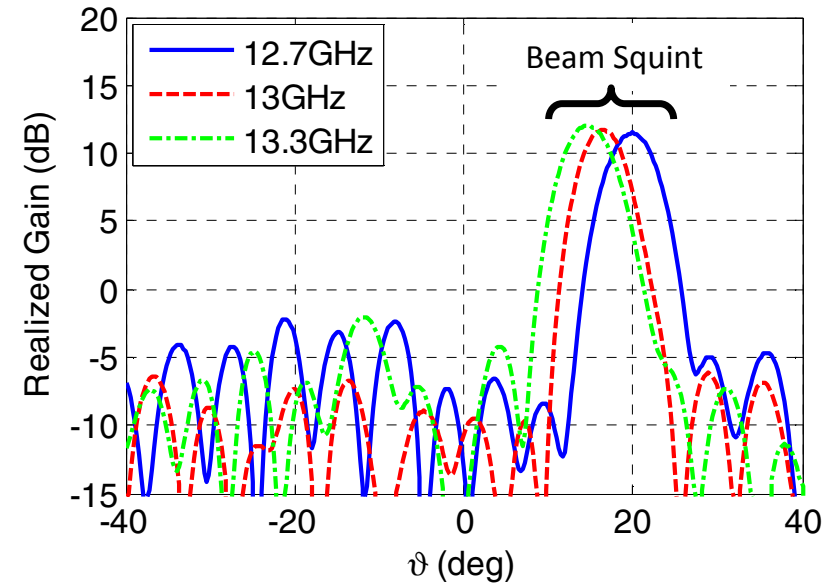

Fig. 18. Measured $\mathrm{g}=35 \mathrm{mil}$ patterns of CPS patch array for a high communications bandwidth

\section{Simulations of Prototype with Fabrication Errors}

During fabrication, to ensure no space between the transmission line traces and the slot edge, the slot was enlarged from the original 50mil width as seen in Figure 19. Due to a manufacturing error, the slot was made overly wide. This meant that when $\mathrm{g}$ was reduced to 0mil, the taper still didn't contact the transmission line traces. Thus, the max achievable propagation constant is significantly reduced. Additionally, the tapered insert was fabricated at an angle of $3.5^{\circ}$ rather than the intended $5^{\circ}$. This introduces extra air to supported wave and thus also reduces the propagation constant for all angles. Figure 20 shows the results accounting for the realities of the design. The only major difference between simulated and measured patterns is gain level. This reduced gain can be attributed to the fractured board. The matching simulation and measurement patterns give validation to the design.

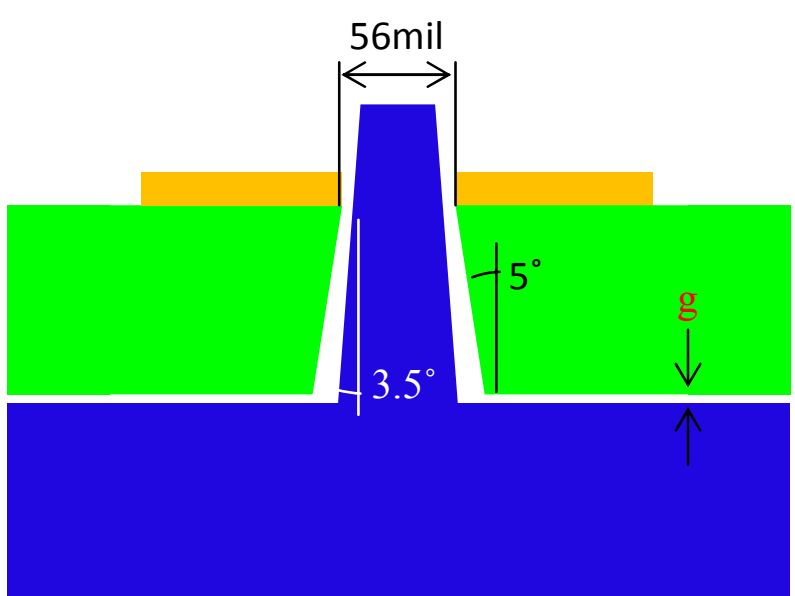

Fig. 19. Transmission line model reflecting various manufacturing errors (insert angle and slot width) 


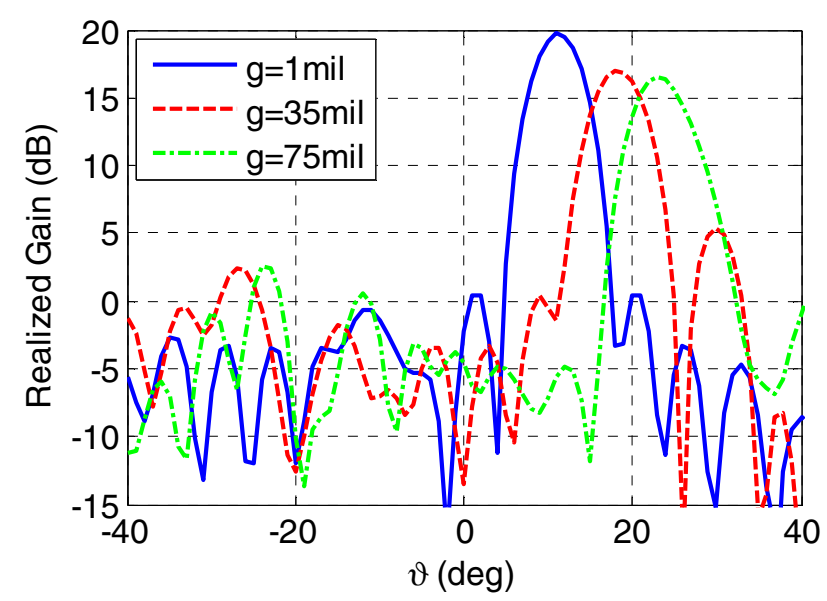

Fig. 20. Simulated result of array with manufacturing errors

\section{CONCLUSION}

A novel 1-D array feeding topology has been presented to reduce cost and complexity of a phased array system. A propagation constant reconfigurable slotted coplanar stripline design was employed to induce beam steering via a small $(\leq 100 \mathrm{mil})$ linear motion, thus eliminating the costly and complex backend including all phase shifters.

Array board material, insert angle $(\alpha)$, and strip spacing $(S)$ were shown to be key factors in determining propagation constant range and thus scan angle range. Meanwhile, trace width $(W)$ is the major determinant of CPS characteristic impedance. This characteristic impedance is found to be a critical aspect to achieve uniform element excitation. By choosing proper line impedance (Eq. 3), array beam steering pattern stability is achieved. This stability increases with more elements.

A 20 element patch array with a TMM4 array board, $\alpha=5^{\circ}, S=50 \mathrm{mil}, W=25 \mathrm{mil}$ was designed to scan from $-25^{\circ} \leq \theta \leq 21^{\circ}$ with mostly uniform gain at $13 \mathrm{GHz}$. A fabricated prototype showed a scan range of $12^{\circ} \leq \theta \leq 25^{\circ}$, a cross-polarization of about $-15 \mathrm{~dB}$ below co-polarization, and a beam squint of $\pm 2.5^{\circ}$ for a bandwidth of $600 \mathrm{MHz}$. Differences from simulated patterns originated from a correctable manufacturing error. Simulations accounting for the discovered fabrication errors compared very well with measured patterns, validating the simulations. This validated concept constitutes a low complexity, low cost alternative to traditional phased arrays.

Future work will allow for better pattern stability through optimized element impedance distributions. Designs will scale to $\mathrm{Ka}$ Band in accordance with satellite communications. Finally, 2-D independent scanning will be accomplished via an extra reconfigurable transmission line feeding an array of 1-D arrays.

\section{ACKNOWLEDGMENT}

This work was supported by a NASA Office of the Chief Technologist's Space Technology Research Fellowship (NSTRF), NASA Grant \#NNX11AN16H. A special thanks to Ms. Elizabeth McQuaid (NASA GRC) and Dr. Kevin Lambert (Vantage Partners, LLC) for their help in the fabrication of the prototype and antenna metrology.

\section{REFERENCES}

[1] Mosca, S.; Ciattaglia, M., "Ant colony optimization applied to array thinning," Radar Conference, 2008. RADAR '08. IEEE , vol., no., pp.1,3, 26-30 May 2008

[2] Schrank, H.; Schuman, H., "Antenna Designer's Notebook-design curves for reducing the number of phase shifters in-phased arrays by subarraying," Antennas and Propagation Magazine, IEEE, vol.35, no.2, pp.56,58, April 1993

[3] N. Host, C-C. Chen, J. Volakis, and F. Miranda, "Novel Phase Array Scanning Using Single Feed Without Using Individual Phase Shifters," $34^{\text {th }}$ Annual Symposium of the Antenna measurement Techniques Association, Oct. 21-26, 2012 Bellevue, WA.

[4] N. Host, C-C. Chen, J. Volakis, and F. Miranda, "Reconfigurable Transmission Line for a Series-Fed Ku-Band Phased Array Using a Single Feed," IEEE Int. Symposium on Antennas and Propagation, July 2013

[5] Mirzaei, H.; Eleftheriades, G.V., "An active artificial transmission line for squint-free series-fed antenna array applications," Microwave Conference (EuMC), 2011 41st European, vol., no., pp.503,506, 10-13 Oct. 2011

[6] Sheikh, S.I.M.; Gibson, A.A.P.; Basorrah, M.; Alhulwah, G.; Alanizi, K.; Alfarsi, M.; Zafar, J., "Analog/Digital Ferrite Phase Shifter for Phased Array Antennas," Antennas and Wireless Propagation Letters, IEEE, vol.9, no., pp.319,321, 2010

[7] Ueda, T.; Yamamoto, S.; Kado, Y.; Itoh, T., "Pseudo-Traveling-Wave Resonator With Magnetically Tunable Phase Gradient of Fields and Its Applications to Beam-Steering Antennas," Microwave Theory and Techniques, IEEE Transactions on, vol.60, no.10, pp.3043,3054, Oct. 2012

[8] Hui, W.W.G.; Bell, J.M.; Iskander, M.F.; Lee, J.J., "Low-Cost Microstrip-Line-Based Ferrite Phase Shifter Design for Phased Array Antenna Applications," Antennas and Wireless Propagation Letters, IEEE, vol.6, no., pp.86,89, 2007

[9] Tang, Hongyan; Donnan, R.; Parini, C., "A novel phase shifter based patch antenna integrated electrostatic peristaltic micropumps," Mobile Technology, Applications and Systems, 2005 2nd International Conference on, vol., no., pp.4 pp.,4, 15-17 Nov. 2005

[10] Tae-Yeoul Yun; Kai Chang, "A low-cost 8 to $26.5 \mathrm{GHz}$ phased array antenna using a piezoelectric transducer controlled phase shifter," Antennas and Propagation, IEEE Transactions on, vol.49, no.9, pp.1290,1298, Sep 2001

[11] Zhi Chang Zheng; Guo Qing Luo, "Design of a compact wideband balun between microstrip and coplanar stripline," Microwave Workshop Series on Millimeter Wave Wireless Technology and Applications (IMWS), 2012 IEEE MTT-S International, vol., no., pp.1,3, 18-20 Sept. 2012 